\title{
Synthesis and characterization of curcumin-phosphatidylcholine complex via solution enhanced dispersion by supercritical $\mathrm{CO}_{2}$
}

\author{
Phan Minh Vuong, Nguyen Minh Ty, Pham Cao Thanh Tung, Phan Thanh Thao* \\ Institute of Chemical Technology, Vietnam Academy of Science and Technology \\ Received 4 April 2017; Accepted for publication 27 August 2017
}

\begin{abstract}
In order to enhance the bioavailability of poorly water-soluble curcumin, solution enhanced dispersion by supercritical carbon dioxide $\left(\mathrm{CO}_{2}\right)$ (SEDS) was employed to prepare curcumin-phosphatidyl choline complex. The reaction parameters were varied and investigated. The typical parameters were determined as follow: $\mathrm{P}=200$ bar, $\mathrm{T}=$ $60{ }^{\circ} \mathrm{C}$, gas flow rate $10 \mathrm{~mL} \cdot \mathrm{min}^{-1}$, molar ratio of phosphatidylcholine:curcumin $2: 1$, ratio of dichloromethane/ $/ \mathrm{scO}_{2} 15$ $\%(\mathrm{w} / \mathrm{w})$. The characteristics of complexation product were measured such as the water solubility, natural stability, UVVis, FTIR and DSC. The FE-SEM image shows that curcumin-phosphatidylcholine complex appeared in two types of spherical shape with the big particle size of complex approximately $0.9 \mu \mathrm{m}$ and nano curcumin with particles size of approximately $100 \mathrm{~nm}$. This study revealed that supercritical $\mathrm{CO}_{2}$ technologies had a great potential in fabricating complex and improving the bioavailability of poorly water-soluble drugs.
\end{abstract}

Keywords. Curcumin, water soluble curcumin, phosphatidylcholine, $\mathrm{CO}_{2}$ supercritical fluids.

\section{INTRODUCTION}

Curcumin (1,7-bis(4-hydroxy-3methoxyphenyl) -16-heptadiene-3,5-dione), derived from the rhizome of the plant Curcuma longa, is a yellow-orange polyphenol compound $[1,2]$. It is widely used as a spice, food preservative, flavoring and coloring agent. Evidence suggests curcumin has the potential to prevent or treat various pathophysiological processes, including cardiovascular disease, carcinogenesis, wound healing and inflammation [1]. However, the low water solubility and low stability are two obstacles that inhibit the high activated property of curcumin [2,5]. Seeking to address these problems, recent studies have explored a number of delivery systems such as micelles, plasma proteins, cyclodextrins, and polymer nanoparticles [3].

Some typical techniques have been used for the preparation of curcumin encapsulated in Phosphatidylcholine (PC), the part of soy lecithin. The PC, an essential part of the cell membrane used in phytosomes technology, acts as a carrier and also nourishes the skin [9]. The curcumin, just like another dietary phenolic, shows polar groups (two phenolic hydroxyls and one enolic hydroxyl) that can interact via hydrogen bonding and polar interactions with complementary groups, like the polar head of PC. The soy lecithin has a highly polarized head, with the negative charge of a phosphate group and the positive charge of the choline ammonium group, and can complex a variety of poorly soluble phenolics, including curcumin [12]. The details of some complexes in the daily dose and specific indications showed higher aqueous and n-octanol solubility and higher antioxidant activity than that of pure curcumin at all dose levels tested [10].<smiles>COc1cc(/C=C/C(=O)CC(=O)/C=C/c2ccc(O)c(OC)c2)ccc1O</smiles>

B<smiles>[R]C(=O)OC(COCP(=O)([O-])OCC[N+](C)(C)C)C([R])=O</smiles>

Figure 1: Structure of (A) curcumin and (B) phosphatidylcholine compounds

However, there are some drawbacks in the complex preparation such as the energy drying and the toxic solvent residues of VOC solvents [6, 9]. In 
this point of view, supercritical $\mathrm{CO}_{2}\left(\mathrm{SCCO}_{2}\right)$ is ideal. Recently, supercritical carbon dioxide $\left(\mathrm{scCO}_{2}\right)$ technologies have shown great potential in the field of particle formation, especially drug micronization. The $\mathrm{scCO}_{2}$ technologies present many advantages, including low critical conditions $(\mathrm{Tc}=304.1 \mathrm{~K}, \mathrm{Pc}=$ $7.38 \mathrm{MPa}$ ), no solvent residue, non-flammability, low cost, and being environmentally benign $[4,7,8]$.

In this work, we used $\mathrm{SCCO}_{2}$ as solvent and solution enhanced dispersion method for the preparation of curcumin-phosphatidylcholine complex. The reaction was conducted in batch condition using high pressure induced autoclave. The reaction parameters such as reactant ratio, reaction temperature, pressure, and reaction time were investigated. The addition of dichloromethane showed significant influence to the reaction yield. UV-Vis spectrometer, HPLC, FTIR, DSC, FE-SEM instruments were used to measure the reaction yield, product solubility, and other properties.

\section{MATERIALS AND METHOD}

\subsection{Materials}

Curcumin (Cur, $96 \%$ ) from Wacker (Germany), phosphatidylcholine (99 \%) from Indian, dichloromethane (HPLC grade, Merck), acetonitrile (HPLC grade, Merck), double distilled de-ion water (DI), $\mathrm{CO}_{2}$ (99.99\%, Air Liquid) were purchased and used as received.

(1) $\mathrm{CO}_{2}$ liquid vessel

(2) Cooler

(3) High pressure pump

(4) Heater exchanger

(5) Magnetic stirrer

(6) Nozzle

(7) High pressure vessel

(8) Valve on/off

(9) Pressure gauges

(10) Backflow valve

(12) Temperature control

(13) Gas flowmeter

\subsection{Synthesis curcumin-phophatidylcholine complex in $\mathrm{CO}_{2}$ media}

The equipment includes a volumetric pump, a 100 $\mathrm{mL}$ stainless steel autoclave laid on a magnetic stirrer, a depressurization system with a two-way coaxial nozzle and a temperature heating. The schematic diagram of the SEDS process is shown in figure 2 . In a typical experiment, $0.48 \mathrm{~g}$ curcumin was dissolved in $50 \mathrm{~mL}$ absolute dichloromethane. $10 \mathrm{~mL}$ of the $20 \%$ phosphatidylcholine dichloromethane solution was dropwise added. The resulting mixture was sonicated at $55 \mathrm{~W}$ for $3 \mathrm{~min}$ to generate the final emulsion then added to the autoclave. The autoclave was closed carefully and connected to the experiment system. The reactor was filled up with $\mathrm{SCCO}_{2}$. The heat was slowly increased, the pressure was fixed and the reaction mixture was kept with continuous stirring by the magnetic stirrer. When the desired experimental conditions were reached, curcumin complex in the autoclave was injected into the high-pressure vessel via a stainless steel coaxial nozzle simultaneously with other supercritical $\mathrm{CO}_{2}$ flow, using a high-pressure liquid pump. During the dispersion process, the precipitation temperature and pressure were maintained as described above. After all the solution was delivered into the high-pressure vessel, the $\mathrm{CO}_{2}$ flow was stopped and the $\mathrm{CO}_{2}$ in the high-pressure vessel was slowly depressurized to atmospheric pressure. The curcumin- phosphatidylcholine complex was then collected on the filter at the bottom of the high-pressure vessel for further characterization.

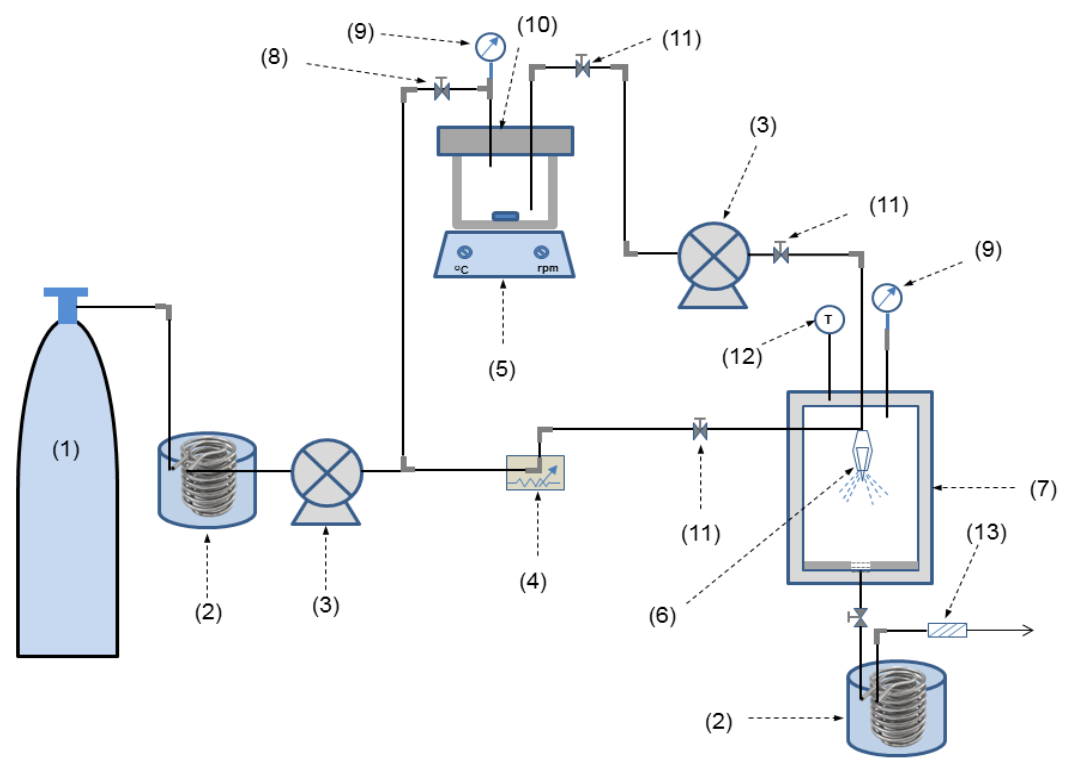

Figure 2: Schematic diagram of the SEDS process 


\subsection{Determination of the reaction yields, solubility, and natural light stability}

The reaction yield was calculated based on the relative amount of insoluble curcumin in product and that in the solid material mixture. For that, 15 $\mathrm{mg}$ of red powder product was dissolved in $10 \mathrm{~mL}$ of DDW with sonication for $30 \mathrm{sec}$. The solution was then centrifuged at 9,000 rpm for $5 \mathrm{~min}$, the supernatant was decanted. This work was repeated 2 more times until no more product be dissolved. The remaining solid in the centrifuge tube is dissolved by acetonitrile/DDW solvent (1:1 volume) mixture. The solution is transferred to the volumetric flask and added solvent to $25 \mathrm{~mL}$. This solution is measured the UV absorption at $425 \mathrm{~nm}$ and calculated the amount based on premade calibration curve.

The reaction yield is calculated based on the equation:

$$
\mathrm{H} \%=\frac{\mathrm{Cur}_{t o l}-\mathrm{Cur}_{f r}}{\mathrm{Cur}_{t o l}} \times 100
$$

with $\mathrm{Cur}_{t o l}$ and $\mathrm{Cur}_{f r}$ are the amount of curcumin in solid material before reaction and amount of curcumin insoluble in the product, respectively.

The water solubility of curcumin in the complex product was measured by dissolving $25 \mathrm{mg}$ of red powder product in $10 \mathrm{~mL}$ of DDW with sonication for $30 \mathrm{sec}$. The solution was then centrifuged at $9,000 \mathrm{rpm}$ for $5 \mathrm{~min}$, the saturation solution was decanted. All clear supernatant solution was collected and added DDW to $25 \mathrm{~mL}$ in a volumetric flask. The measurement was conducted on HPLC equipped with a Zorbax Eclipse Plus C18 (4.6 x 250, $5 \mu \mathrm{m})$ column using DAD detector and the amount of curcumin is calculated based on the premade calibration curve. The measurents concentration of curcumin in saturation solution under sun light at room temperature in one week was conduct by UV absorption at $425 \mathrm{~nm}$ based on premade calibration curve.

\subsection{Characterisation of curcumin- phosphatidylcholine complex}

\subsubsection{Thermal analysis}

Thermal analysis was carried out on about $3 \mathrm{mg}$ sample by using a Differential Scanning Calorimetry (DSC, Model 204 F1 Phoenix, NETZSCH, Germany) from 40 to $220{ }^{\circ} \mathrm{C}$ at a heating rate of 5 ${ }^{\circ} \mathrm{C} /$ min under an $\mathrm{N}_{2}$ gas stream.

\subsubsection{FTIR spectroscopy}

Study of the complex product was conducted with FTIR Tensor 27, Brucker, Germany spectrophotometer. The samples were combined with potassium bromide, and the mixtures of the samples and potassium bromide were pressed into a transparent tablet. The FTIR spectra of the samples were recorded with the wave number ranging from 4,000 to $400 \mathrm{~cm}^{-1}$.

\subsubsection{FE-SEM}

The surface morphology of curcumin nanoparticles was visualized by scanning electron microscopy (FE-SEM) (JSM-7401F, JEOL, Tokyo, Japan). These samples were then gold sputter-coated under argon prior to imaging. Complex sample was made conductive by sputtering a thin layer of gold onto their surface. The particle size and particle size distribution of the samples were analyzed by Analysis Station JED-2300 software from the SEM photographs.

\section{RESULTS AND DISCUSSION}

\subsection{Influence of reaction parameters on yield of product}

The influence of temperature on the reaction was investigated and shown in Fig. 4A. The reaction yield increased with increasing the temperature. However, when further increased the reaction temperature more than $60{ }^{\circ} \mathrm{C}$, reaction yield decreased. For the other later investigations, we choose the reaction temperature at $60{ }^{\circ} \mathrm{C}$. Pressure built up inside the reactor is due to the occupancy of $\mathrm{SCCO}_{2}$ in all the reactor volume. The pressure rapidly increased when increasing the temperature. Influence of pressure to reaction yield was shown in Fig. 4B. When carried out the reaction without using $\mathrm{SCCO}_{2}$ and the reaction temperature at $60{ }^{\circ} \mathrm{C}$, we did not observed the indication of pressure on pressure gauge. At the same time, the yield of reaction was rather low. In the presence of $\mathrm{SCCO}_{2}$, the reaction yield dramatically increased. However, when the pressure was controlled in the range of 30 to 300 bar the reaction yield slightly increased. We used the pressure of 200 bar for all other later studies. The reaction time effect is shown in Fig. 4F. Interestingly the complexation occurred and got the yield up to $82 \%$ within the first hour of reaction time. With keeping the reaction time longer, reaction yield increased slowly. The highest yield was obtained at $99.8 \%$ after 3 hours. The relationship of curcumin and phosphatidylcholine molar ratio in the starting materials with the reaction yield is shown in 
Fig. 4D. The highest yield was obtained at the ratio of $1: 2$. When we kept increasing the amount of phospholipid in the ratio from 2.0 to 3.0, the yield almost did not increase further. In this work, the use of supercritical $\mathrm{CO}_{2}$ as an solution enhancing dispersion showed strong positive effect. In this case, the reaction yield dramatically increased to $84.5 \%$ compared to $65.6 \%$ in case of without using $\mathrm{scCO}_{2}$. The investigation results are shown in Fig. 4C. The highest effect is shown at the gas flow rate $10 \mathrm{~L} / \mathrm{min}$. The yield even decrease if increase gas flow rate.
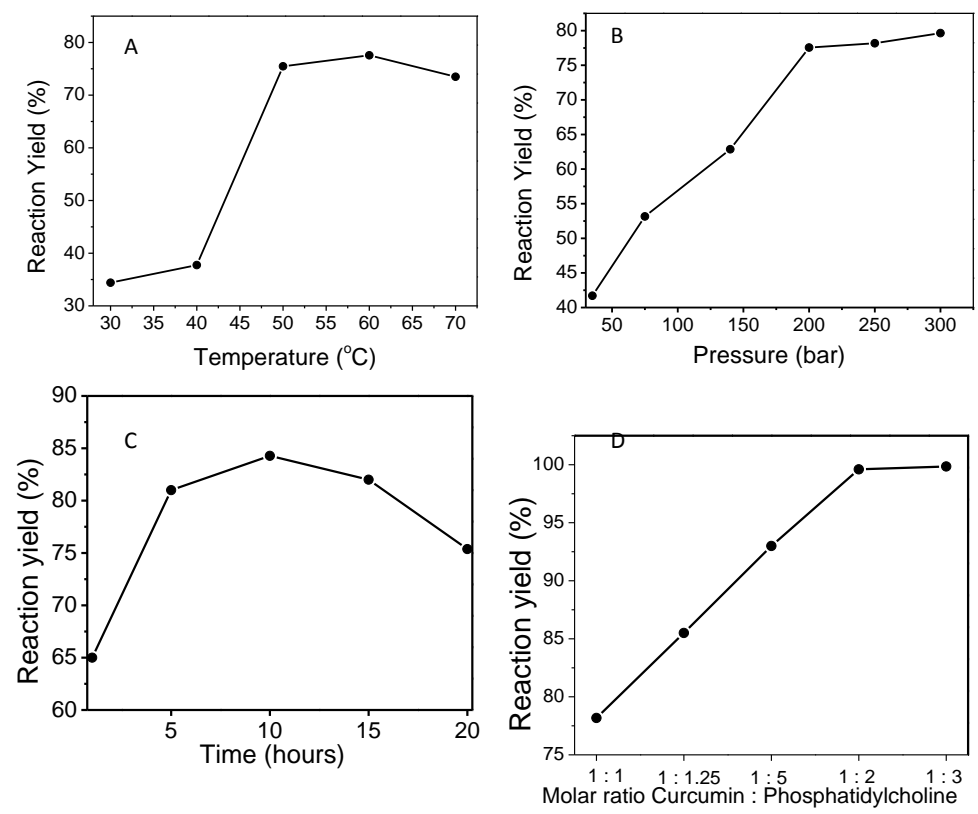

$E$
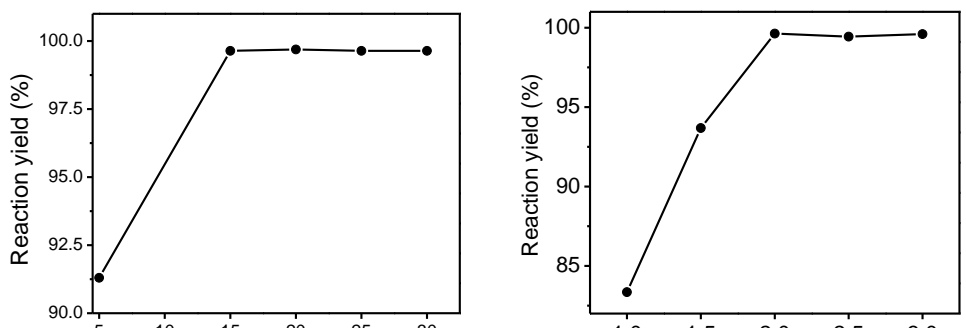

Figure 4: The influences of reaction yield by the reaction condition such as temperature (A), pressure (B), gas flow rate (C), the molar ratio of phosphatidylcholine with respect to curcumin (D), the ethanol amount $(\mathrm{E})$ and reaction time $(\mathrm{F})$

The influence of dichloromethane in the reaction is shown in Fig. 4E. By physical mixing and the dichloromethane excited as absorbed dichloromethane in phosphatidylcholine, the encapsulate almost did not occur. When dichloromethane added, the reaction yield dramatically increased and got the highest of $99.4 \%$ with ratio ${ }^{\mathrm{m}}$ dichloromethane $/{ }^{\mathrm{m}} \mathrm{CO}_{2}(15 \%)$ was added into the reactant mixture. With the addition of dichloromethane, it probably partially dissolved the surface of reactants and increased the reaction on the fresh surface. Moreover, the dichloromethane soluble product of this surface reaction easily diffused out of surface and enhanced the surface dissolving. As the result of this interaction, the equilibrium of this reaction shifted toward the complexation formation direction.

\subsection{Solubility and natural light stability}

The improvement of solubility of curcumin complexation product in water is the main goal to be achieved. As the result of the investigation, the solubility of curcumin is obtained $198 \mu \mathrm{g} / \mathrm{mL}$ in DI water corresponding to $2.5 \times 10^{-4} \mathrm{~mol} / \mathrm{L}$ while we observed only the trace amount of free curcumin in DI water. Based on the calculation, the water solubility of complexed curcumin is approximately 4000 times enhanced compared to that of intrinsic curcumin. High stability of the curcumin as shown 
in the sample of the complex indicating a very good, after 3 days, the concentration of curcumin retention was $192 \mu \mathrm{g} / \mathrm{mL}$, corresponds $96.9 \%$.

Continued survey to after one week, curcumin retention was $93.5 \%$. By on the facts about reaction yield and stability of the complex, it shown the role of phosphatidylcholine with complex system. Almost all of phospholipid and phosphatidylcholine can form micelles with curcumin or another drug, and enhance solubility and stability of them $[9,10]$.

\subsection{UV-Vis absorption spectrum}

The complexation obviously changed the solubility as well as the spectrum of product with respect to that of free curcumin. Fig. 6A-B show the photograph pictures of phosphatidylcholine and complexation product in DDW. Free curcumin is clearly insoluble. In contrast, complexation product in DDW is quickly dissolved and formed a clear yellow color solution as shown in Fig. 6B. Consistent with these images, the UV-Vis absorption spectrum of product in Fig. 6C clearly showed the characteristic absorption peak of curcumin at 425 $\mathrm{nm}$.

\subsection{DSC}

DSC thermograms of each starting material, physical mixing sample, and complexation product are shown and indicated in Fig.5. The thermogram curve of phosphatidylcholine showed the endothermal peak starting from $150{ }^{\circ} \mathrm{C}$ to $170{ }^{\circ} \mathrm{C}$. While the endothermal peak of free curcumin appeared a sharp peak at $175-178{ }^{\circ} \mathrm{C}$ as the melting point and high crystallinity. We did not observe the peak as the absorbed water loss in both cases. In the case of physical mixing sample, two peaks in the region $150-164{ }^{\circ} \mathrm{C}$ and $174-178^{\circ} \mathrm{C}$ corresponding to phosphatidylcholine and curcumin respectively were absorbed. With the complexation product, this thermogram is almost flattened, and the endothermal peak due to the molten curcumin disappeared. This result reveals that the initial crystalline structure of curcumin was collapsed. In the molecular level, curcumin molecules in the product are disorder arrangement. Based on DSC characterizations, it is high possibility that curcumin was encapsulated in the phospholipid micelles.

\subsection{FTIR spectroscopy}

Figure 7 represents a graph of the FTIR analysis of the curcumin, phosphatidylcholine, physical mixing, and complex. In the spectra of free curcumin and

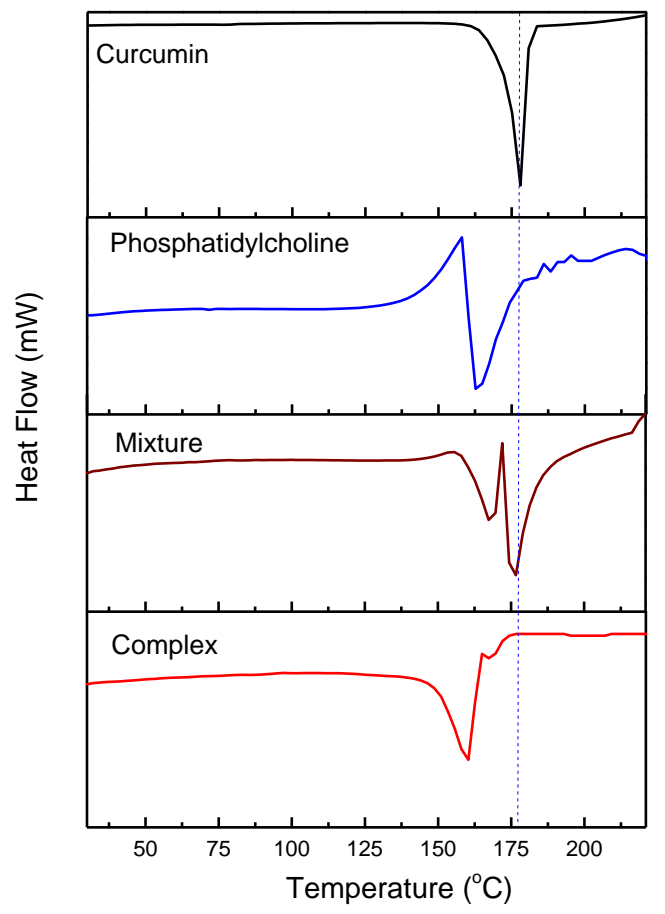

Figure 5: The DSC curve of curcumin, phosphatidylcholine, phosphatidylcholine physical mixing and complex as indicated
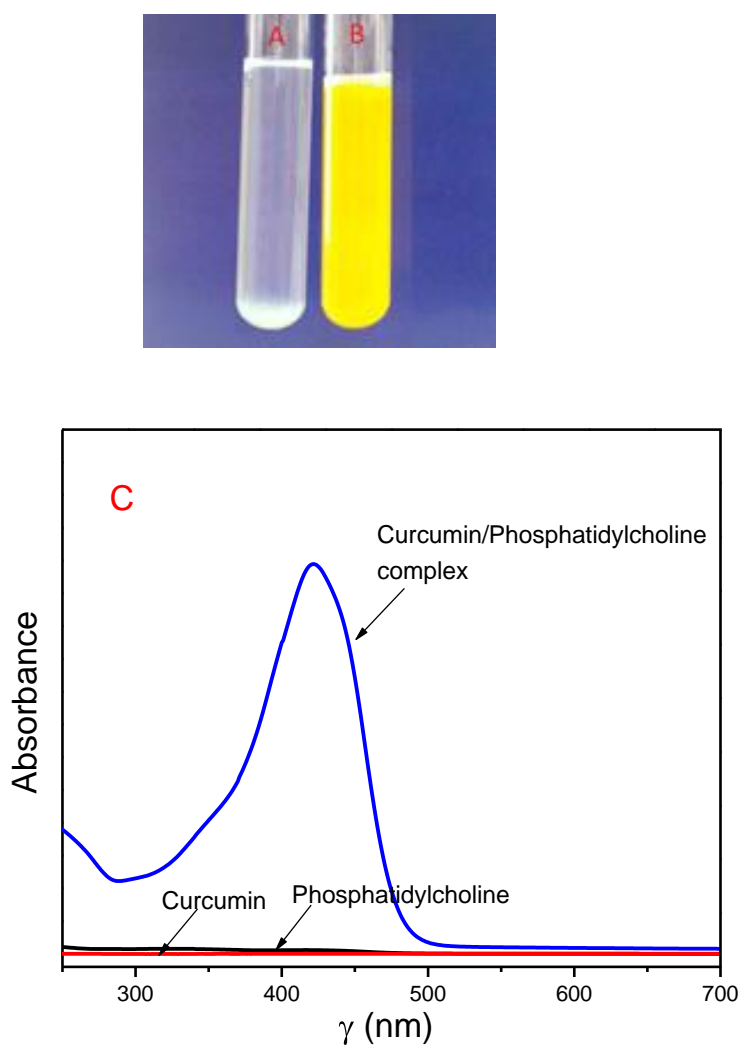

Figure 6: The photograph pictures of phosphatidylcholine in water (A), complex in water (B), the UV-Vis absorption spectra of complex, curcumin free and phosphatidylcholine in $\mathrm{H}_{2} \mathrm{O}$ as indicated $(\mathrm{C})$ 
physical mixing sample, the vibration peaks at 1628 and $1601 \mathrm{~cm}^{-1}$ corresponding to the $\mathrm{C}=\mathrm{O}$, and $\mathrm{C}=\mathrm{C}$ vibration mode of aromatic ring respectively are quite sharp. However, those of peaks are broader and lower intensity in both the case of $\mathrm{Cur} / \mathrm{PC}$ complex. The vibration peak of $\mathrm{C}=\mathrm{C}$ of the aromatic ring in curcumin and physical mixing at $1628 \mathrm{~cm}^{-1}$ shifted to $1631 \mathrm{~cm}^{-1}$, peak $1601 \mathrm{~cm}^{-1}$ shifted to 1588 $\mathrm{cm}^{-1}$ in case of complex $[13,14]$. The vibration peak of $\mathrm{C}=\mathrm{O}$, and $\mathrm{C}-\mathrm{C}=\mathrm{O}$ in physical mixing at $1510 \mathrm{~cm}^{-}$ ${ }^{1}$ shifted to $1515 \mathrm{~cm}^{-1}$, the intensity of this peak decreased obviously. The peak at $1280 \mathrm{~cm}^{-1}$ due to

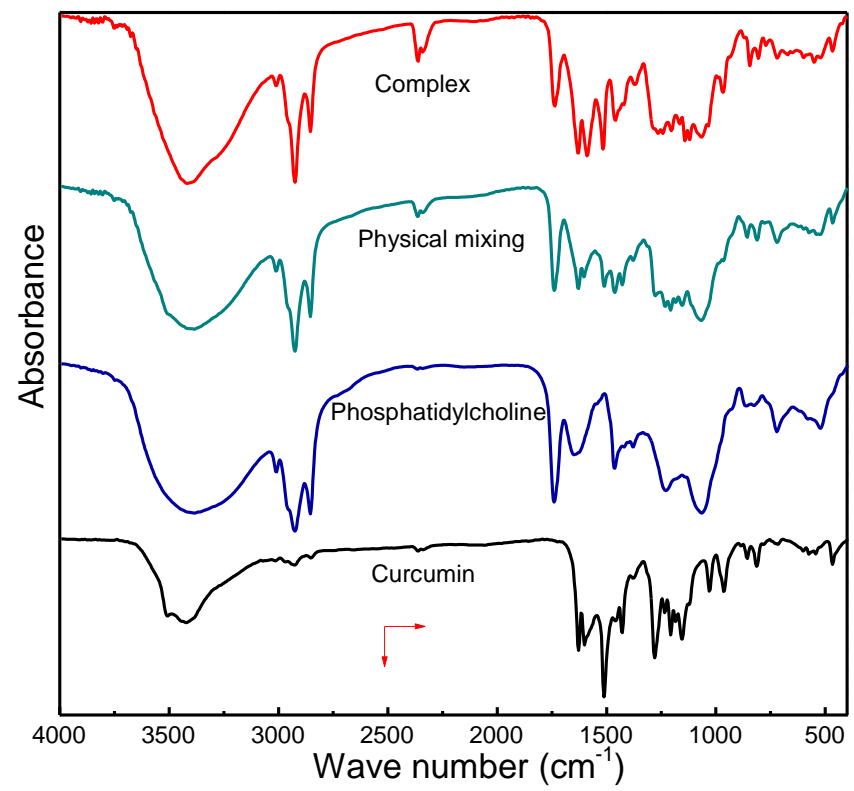

vibration mode of $\mathrm{C}=\mathrm{C}-\mathrm{H}$ and aromatic $\mathrm{C}-\mathrm{O}$ stretching are clearly seen in the spectra of curcumin and physical mixing shifted to $1264 \mathrm{~cm}^{-1}$, suggesting that the interaction to one or both phenol group with phosphatidylcholine occurred in complex formation. The peak at $1228 \mathrm{~cm}^{-1}$ corresponding to antisymmetric $\mathrm{PO}_{2}^{-}$stretching provides information about the interfacial region of the phospholipid splitting into two separate bands at 1242 and 1203 $\mathrm{cm}^{-1}$ and lower intensity in spectrum of complex [9]. This results indicate when cucumin molecule was encalsulated in phosphatidylcholine there is

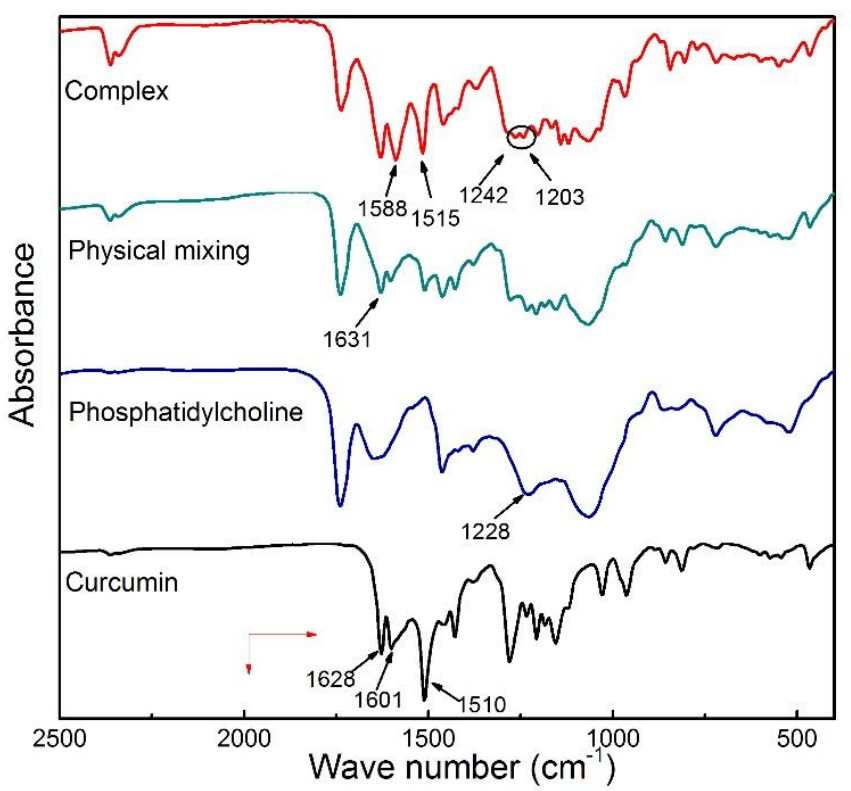

Figure 7: The FTIR absorption spectra of curcumin-phosphatidylcholine complex, physical mixing, curcumin free and phosphatidylcholine as indicated
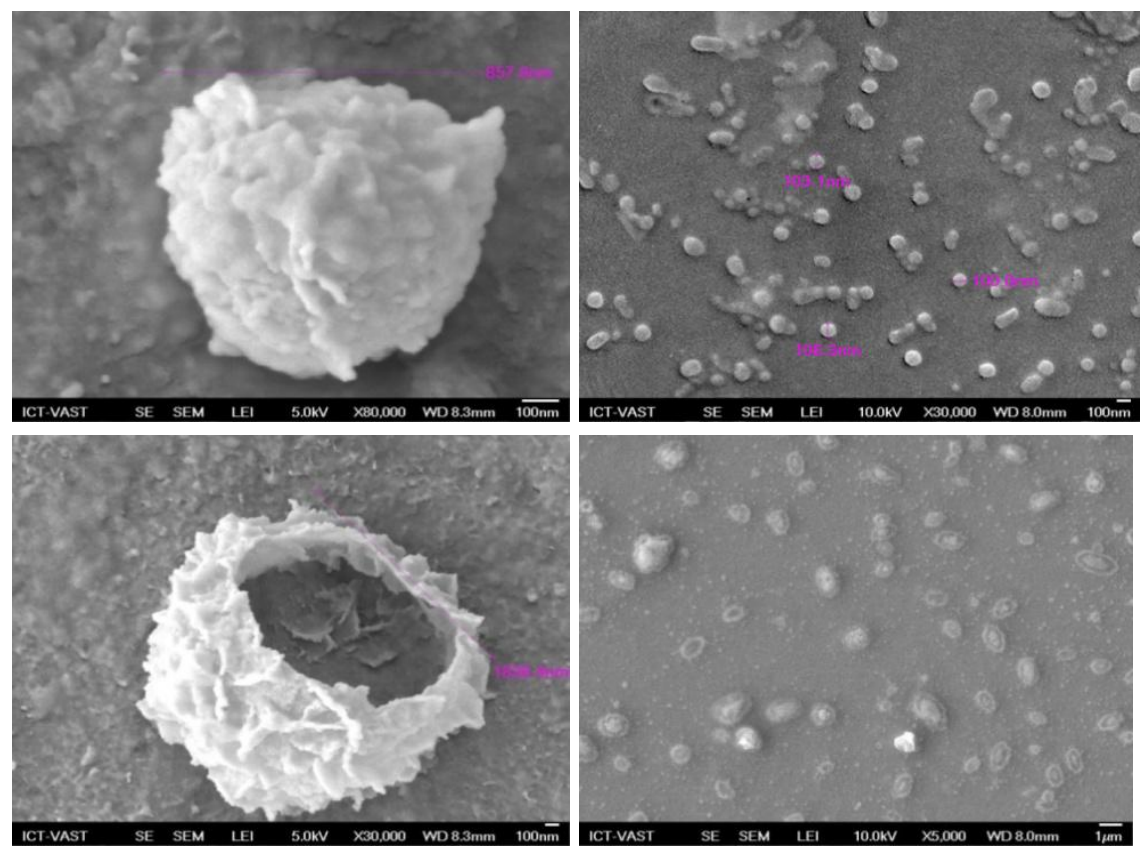

Figure 8: FE-SEM image of curcumin-phosphatidylcholine complex 
interaction of curcumin with $\mathrm{PO}_{2}$-group of phosphatidylcholine membrane for formation phytosome structures [11].

\subsection{FE-SEM}

Figure 8 shown the SEM micrographs of the curcuminphosphatidylcholine complex fabricated by the SEDS process. It can be concluded that phytosomes possessed an irregular spherical morphology with a mean particle size from 857 to $1,824 \mathrm{~nm}$.

Moreover, in the complex there is the presence of curcumin nanoparticles with particles size approximately $100 \mathrm{~nm}$. FE-SEM image indicates, that curcumin was encapsulated on phosphatidylcholine membrane and phytosome structure was formed. In liposome structure the active principle is dissolved in an internal pocket or floats in the layer membrane, while in phytosomes the active principle is anchored to the polar head of phospholipids, becoming an integral part of the membrane [9]. Evidently, the SEDS process is an effective way to prepare curcumin nanoparticles. Moreover, by adjusting the different process parameters of the SEDS process, curcumin nanoparticles with controllable particle size can be prepared successfully $[7,8]$.

\section{CONCLUSIONS}

In this work, we have reported the research result in the preparation of complex curcuminphosphatidylcholine. The reaction is conducted in the $\mathrm{SCCO}_{2}$ condition at $\mathrm{P}=200$ bar, $\mathrm{T}=60{ }^{\circ} \mathrm{C}$, gas flow rate $10 \mathrm{~mL} \cdot \mathrm{min}^{-1}$, molar ratio of phosphatidylcholine:curcumin $2: 1$, ratio of dichloromethane $/ \mathrm{scCO}_{2} 10 \%(\mathrm{w} / \mathrm{w})$ temperature 60 ${ }^{\circ} \mathrm{C}$, to reach high inclusion yield. The complexation product is highly soluble in water and high potential to bring to practical application.

The morphology of complex was determined by FE-SEM. According the FE-SEM image, almost of phosphatidylcholine molecules were in form of complex with cucumin. FTIR results showed there was interaction of curcumin molecule to $\mathrm{PO}^{2-}$ group of phosphatidylcholine membrane for phytosomes formation.

Acknowledgement. This work was supported by Vietnam Academy of Science and Technology (VAST), grant number VAST.CTG.07/13-14.

\section{REFERRENCES}

1. Glen R. B. Irving, Ankur Karmokar. Curcumin: The potential for efficacy in gastrointestinal diseases, Best Practice \& Research Clinical Gastroenterology, 25, 519-534 (2011).

2. Murali M. Y., Meena J., Subhash C.C. Curcumin nanoformulations: a future nanomedicine for cancer, Drug Discovery Today, 17(1-2), 71-80 (2012).

3. Takaaki Harada, Duc-Truc Pham, Stephen F. Lincoln, Tak W. Kee. The Capture and Stabilization of Curcumin Using Hydrophobically Modified Polyacrylate Aggregates and Hydrogels, J. Phys. Chem. B., 118, 9515-9523 (2014).

4. P. Munshi, S. Bhaduri. Supercritical $\mathrm{CO}_{2}:$ a twentyfirst century solvent for the chemical industry, Current Science, 97, 63-72 (2009).

5. Bhawana, K. B Rupesh, S. B Harpreet, V. K. Jain, J. Nidhi. Curcumin Nanoparticles: Preparation, Characterization, and Antimicrobial Study, J. Agric. Food Chem., 59, 2056-2061 (2011).

6. Xiaoxue Zhang, Saar a Heinonen and Erkki Levanen. Applications of supercritical carbon dioxide in materials processing and synthesis, RSC Adv., 4, 61137-61152 (2014).

7. M. Turk, P. Hils, B. Helfgen, M. A. Wahl. Micronization of pharmaceutical substances by the Rapid Expansion of Supercritical Solutions (RESS): a promising method to improve bioavailability of poorly soluble pharmaceutical agents, Journal of Supercritical Fluids, 22, 75-84 (2002).

8. Mahshid Kalani, Robiah Yunus. Application of supercritical antisolvent method in drug encapsulation: a review, International Journal of Nanomedicine, 6, 1429-1442 (2011).

9. Jagruti Patel, Rakesh Patel, Kapil Khambholja, Nirav Patel. An overview of phytosomes as an advanced herbal drug delivery system, Asian Journal of Pharmaceutical Sciences, 4(6), 363-371 (2009).

10. Parneet Kaur, Parul Sen, Dr Sandeep Arora, Arvind Sharma. Emerging Trends and Future Prospective of Phytosomes as Carrier for Enhanced Bioavailabilty of Bioactives: A Review, PhTechMed., 1(3) (2012).

11. Süleymanoglu E. The use of ir spectroscopy after rehydration to follow ternary lipoplex formation and design as a metal-based dna nanopharmaceuticals, Sec. Biol. Med. Sci., 61-80 (2009).

12. Kidd P. M. Bioavailability and Activity of Phytosome Complexes from Botanical Polyphenols: The Silymarin, Curcumin, Alt. Med. Rev., 14, 226246 (2009).

13. Mohan P. R. K., Sreelakshmi G., Muraleedharan C. V., Roy J. Water soluble complexes of curcumin with cyclodextrins: Characterization by FT-Raman spectroscopy, Vibra. Spectro., 62, 77-84 (2012).

14. Bo T., Li M., Huai-You W., Guo-Ying Z. Study on the Supramolecular Interaction of Curcumin and $\beta$ cyclodextrin by Spectrophotometry and Its Analytical Application, J. Agric. Food Chem., 50, 1355-1361 (2002). 
